\title{
A model predictive controller for robots to follow a virtual leader Dongbing $\mathrm{Gu}^{*}$ and Huosheng $\mathrm{Hu}$
}

\author{
School of Computer Science and Electronic Engineering, University of Essex, UK
}

(Received in Final Form: November 19, 2008)

\begin{abstract}
SUMMARY
In this paper, we develop a model predictive control (MPC) scheme for robots to follow a virtual leader. The stability of this control scheme is guaranteed by adding a terminal state penalty to the cost function and a terminal state region to the optimization constraints. The terminal state region is found by analyzing the stability. Also a terminal state controller is defined for this control scheme. The terminal state controller is a virtual controller and is never used in the control process. Two virtual leader-following formation models are studied. Simulations on different formation patterns are provided to verify the proposed control strategy.
\end{abstract}

KEYWORDS: Control of robotic systems; Mobile robots; Multi-robot systems; Robot dynamics; Navigation.

\section{Introduction}

Formation control has been one of the important research topics in multiple robot systems as it is applicable to many areas such as geographical exploration, rescue operations, surveillance, mine sweeping, and transportation. ${ }^{1-5}$

Different approaches have been developed recently, for example, behavior-based control, ${ }^{6,7}$ LQ control, ${ }^{8}$ visual servoing control, ${ }^{9}$ Lyapunov-based control, ${ }^{10}$ input and output feedback linearization control, ${ }^{11,12}$ graph theory, ${ }^{13,14}$ and nonlinear control. ${ }^{15}$

A model predictive control (MPC) is one of the frequently applied advanced control techniques in the industry. It was shown that using an infinite receding horizon can guarantee MPC control stability for even nonlinear systems, ${ }^{16}$ but it is computationally intractable in practice. For a finite receding horizon, it was proved that the stability can be guaranteed by forcing the terminal state to equal to zero. ${ }^{17}$ Further work shows that the terminal state equality constraint can be relaxed as a terminal state inequality, i.e., a terminal state region, by adding a terminal state penalty to the cost function. ${ }^{18,19}$ Recently, the researches in refs. [20-24] show that nonlinear controllers can be used to find the terminal state region as long as a stability condition is met. The works in refs. [21, 23] show that the terminal state penalty can be a control Lyapunov function that will guarantee the stability once the terminal state is within the terminal state region.

The advantages of using an MPC compared to using other control approaches are that it can solve constrained control problems and optimize control performance. Applying an MPC to formation control has been reported in refs. [25, 26]. In ref. [26], a dual mode MPC was used for robot formation.

\footnotetext{
* Corresponding author. E-mail: dgu@essex.ac.uk
}

To secure the stability, the dual mode controller has to switch from an MPC control to a terminal state controller. The switching would result in unsmoothed control signals. The problem of applying an MPC to formation control lies in the states coupling in the cost function because each robot needs its neighbors' state to predict its future states and control signals. In ref. [25], a distributed MPC was applied to the virtual leader formation control for linearized models, in which the center point of a team formation was treated as the virtual leader. By adding a so-called compatibility constraint, the MPC can handle the coupled states in the cost function. The compatibility constraint enforces a degree of consistency between what a robot plans to do and what its neighbors believe that it will plan to do. The MPC used in ref. [25] was proposed in ref. [18].

The formation control where a robot or two robots follow a virtual leader is a different formation control strategy and was formulated as two models: $l-\phi$ control and $l-l$ control. ${ }^{11,12,27,28}$ Due to the use of local information in these two models, the MPC control has a potential to be implemented in a distributed mode. This is crucial for the MPC which requires heavy computation ability.

In this paper, we investigate how to apply MPC to the leader-following formation control with the nonlinear kinematic model. Specifically, the $l-\phi$ and $l-l$ control models are adopted in this paper. Although the general M PC with a terminal state controller has been used before, a terminal state controller for the formation control problems in this paper has not been developed so far. The formation control of two-wheel robots has strong nonlinearity and it is not trivial to apply the general theory. The paper mainly focuses on finding a better terminal state controller and its corresponding terminal region. To do so, a Lyapunov-like function is developed as the terminal state penalty for the MPC stability and a terminal state controller is designed. The terminal state controller is never applied to control the robots. Based on the Lyapunov-like function and the terminal state controller, a new terminal state region is found. This terminal state region is used to constrain the MPC optimization.

The paper is organized as follows. Section 2 introduces the leader-following formation models. The MPC control framework is described in Section 3. The terminal state controller and terminal state region are discussed in Section 4. Simulation results are provided in Section 5. Finally, our conclusion and future works are discussed in Section 6.

\section{Leader-following Formation Models}

A differential driving mobile robot has two rear driving wheels and a front castor for body support. The speed control 


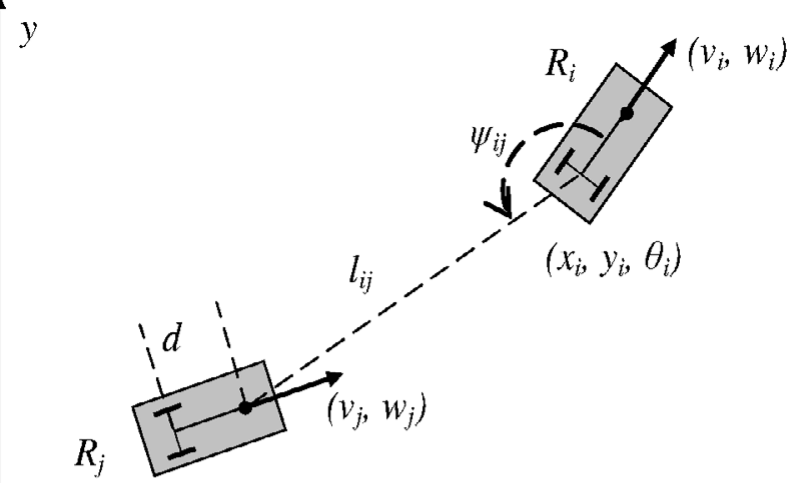

$\left(x_{j}, y_{j} \theta_{j}\right)$

(a) $l-\phi$ control model

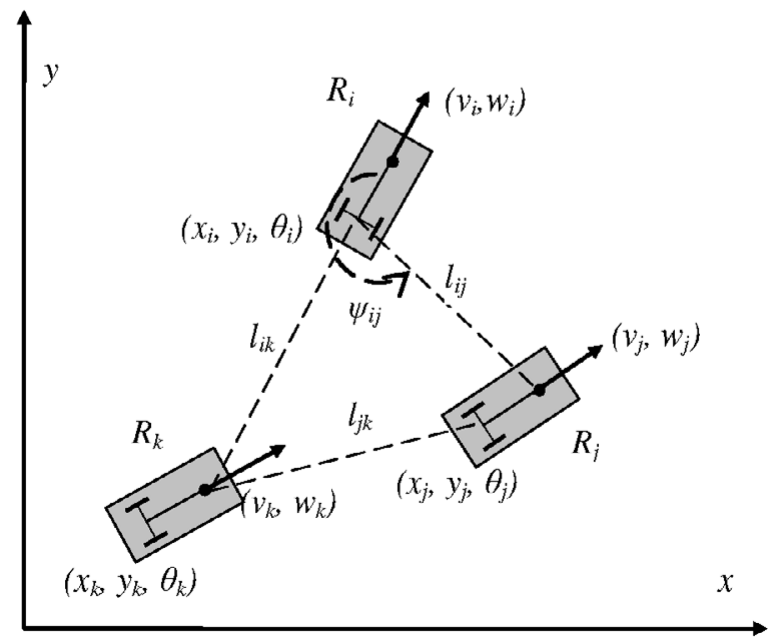

(b) $l-l$ control model

Fig. 1. Leader-following models.

of the two rear wheels leads to the control of linear speed and angular speed. The kinematics equation is

$$
\left[\begin{array}{c}
\dot{x}_{i} \\
\dot{y}_{i} \\
\dot{\theta}_{i}
\end{array}\right]=\left[\begin{array}{cc}
\cos \theta_{i} & 0 \\
\sin \theta_{i} & 0 \\
0 & 1
\end{array}\right]\left[\begin{array}{c}
v_{i} \\
w_{i}
\end{array}\right],
$$

where $\mathbf{x}_{i}=\left[x_{i}, y_{i}, \theta_{i}\right]^{T}$ is the position and orientation of a mobile robot $R_{i}$ and $\mathbf{u}_{i}=\left[v_{i}, w_{i}\right]^{T}$ is its speed control signals.

The leader-following control can be formulated in two models as shown in Fig. $1 .{ }^{27}$ Figure 1(a) is an $l-\phi$ control model where the formation pattern is specified by the separate distance $l_{i j}$ and the relative bearing $\psi_{i j}$ for two robots $R_{i}$ and $R_{j}$. The desired formation pattern can be defined as the desired separate distance $l_{i j}^{d}$ and the relative bearing $\psi_{i j}^{d}$. The follower $R_{j}$ regulates the formation state errors $\mathbf{z}_{j}$ of the separate distance and the relative bearing through its speed control signals $\mathbf{u}_{j}=\left[v_{j}, w_{j}\right]^{T}:$

$$
\mathbf{z}_{j}=\left[\begin{array}{c}
\tilde{l}_{i j} \\
\tilde{\psi}_{i j}
\end{array}\right]=\left[\begin{array}{c}
l_{i j}^{d} \\
\psi_{i j}^{d}
\end{array}\right]-\left[\begin{array}{c}
l_{i j} \\
\psi_{i j}
\end{array}\right] .
$$

The formation control can be investigated by modeling the formation state error $\mathbf{z}_{j}$ as follows:

$$
\begin{aligned}
\dot{\mathbf{z}}_{j} & =-G_{j} \mathbf{u}_{j}+F_{j} \mathbf{u}_{i}, \\
\dot{\phi}_{i j} & =w_{i}-w_{j},
\end{aligned}
$$

and

$$
\begin{aligned}
G_{j} & =\left[\begin{array}{cc}
\cos \left(\phi_{i j}+\psi_{i j}\right) & d \sin \left(\phi_{i j}+\psi_{i j}\right) \\
-\frac{\sin \left(\phi_{i j}+\psi_{i j}\right)}{l_{i j}} & \frac{d \cos \left(\phi_{i j}+\psi_{i j}\right)}{l_{i j}}
\end{array}\right], \\
F_{j} & =\left[\begin{array}{cc}
\cos \psi_{i j} & 0 \\
-\frac{\sin \psi_{i j}}{l_{i j}} & 1
\end{array}\right],
\end{aligned}
$$

where $\phi_{i j}=\theta_{i}-\theta_{j}$ and $d$ is the distance between the robot position $\left(x_{i}, y_{i}\right)$ and the robot hand position as shown in Fig. 1.

Figure 1(b) is an $l-l$ control model, in which $R_{i}$ is the leader and $R_{j}$ and $R_{k}$ are the followers. The formation pattern is specified by the relative bearing $\psi_{i j}$ in the coordinate form of the leader and the separate distances $l_{i j}, l_{i k}$, and $l_{j k} .{ }^{27}$ The followers can use their speed control signals $\mathbf{u}_{j k}=\left[v_{j}, w_{j}, v_{k}, w_{k}\right]^{T}$ to regulate the formation state error $\mathbf{z}_{j k}$ :

$$
\mathbf{z}_{j k}=\left[\begin{array}{c}
\tilde{l}_{i j} \\
\tilde{\psi}_{i j} \\
\tilde{l}_{i k} \\
\tilde{\psi}_{i k}
\end{array}\right]=\left[\begin{array}{c}
l_{i j}^{d} \\
\psi_{i j}^{d} \\
l_{i k}^{d} \\
\psi_{i k}^{d}
\end{array}\right]-\left[\begin{array}{c}
l_{i j} \\
\psi_{i j} \\
l_{i k} \\
\psi_{i k}
\end{array}\right] .
$$

The formation state error $\mathbf{z}_{j k}$ can be formulated as follows:

$$
\begin{aligned}
\dot{\mathbf{z}}_{j k} & =-G_{j k} \mathbf{u}_{j k}+F_{j k} \mathbf{u}_{i}, \\
\dot{\theta}_{j} & =w_{j}, \dot{\theta}_{k}=w_{k},
\end{aligned}
$$

and

$$
\begin{aligned}
G_{j k} & =\left[\begin{array}{cccc}
\cos \gamma_{i j} & d \sin \gamma_{i j} & 0 & 0 \\
-\frac{\sin \gamma_{i j}}{l_{i j}} & \frac{d \cos \gamma_{i j}}{l_{i j}} & 0 & 0 \\
0 & 0 & \cos \gamma_{i k} & d \sin \gamma_{i k} \\
-\cos \psi_{j k} & 0 & \cos \gamma_{j k} & d \sin \gamma_{j k}
\end{array}\right], \\
F_{j k} & =\left[\begin{array}{cc}
\cos \psi_{i j} & 0 \\
-\frac{\sin \psi_{i j}}{l_{i j}} & 1 \\
\cos \psi_{i k} & 0 \\
0 & 0
\end{array}\right],
\end{aligned}
$$


where $\gamma_{i j}=\theta_{i}-\theta_{j}+\psi_{i j}$ and $\gamma_{j k}=\theta_{j}-\theta_{k}+\psi_{j k}$. It should be noted that the states and the inputs of the leader are known to the followers. They are assumed to be bounded and treated as time-varying disturbances to the systems.

\section{Stabilizing MPC Control}

A nonlinear nominal control system without considering any uncertainties, like Eqs. (3) and (6), can be generally expressed as follows:

$$
\dot{\mathbf{z}}(t)=\mathbf{f}(t, \mathbf{z}(t), \mathbf{u}(t)),
$$

where $\mathbf{z}(t) \in R^{n}$ and $\mathbf{u}(t) \in R^{m}$ are the $n$-dimensional error state and $m$-dimensional control vector, respectively. For the two formation models mentioned above, $\mathbf{z}(t)$ represents $\mathbf{z}_{j}(t)$ or $\mathbf{z}_{j k}(t)$ and $\mathbf{u}(t)$ represents $\mathbf{u}_{j}(t)$ or $\mathbf{u}_{j k}(t)$.

The MPC control is to find a suitable $\mathbf{u}(t)$ to drive the system (8) to move toward the equilibrium $(\mathbf{z}(t)=0$ and $\mathbf{u}(t)=0)$. The constraints normally used are the control signal saturation constraints that can be expressed as

$$
\mathbf{u}(t) \in U,
$$

where $U$ is a compact and convex set.

The cost function for the leader-following formation control can be defined as follows:

$J(t, \mathbf{z}(t), \mathbf{u}(t))=g(\mathbf{z}(t+T))+\int_{t}^{t+T} L(\tau, \mathbf{z}(\tau), \mathbf{u}(\tau)) \mathrm{d} \tau$,

where $L(t, \mathbf{z}(t), \mathbf{u}(t))=\mathbf{z}(t)^{T} P \mathbf{z}(t)$. Here $P$ is a positive definite symmetric weight matrix, $T$ is the predictive control horizon, $g(\mathbf{z}(t))$ is assumed to be a continuous, differentiable function, $g(0)=0$, and $g(\mathbf{z}(t))>0$ for all $\mathbf{z}(t) \neq 0$.

The MPC stability can be guaranteed by using a terminal state penalty term $g(\mathbf{z}(t))$ in the cost function and adding a terminal state constraint $\Omega$ to the optimization problem (OP). We assume the time interval of the MPC control to be $\delta$. At time $t$, the open loop OP in the MPC control framework to be solved online can be formulated as

$$
\min _{\mathbf{u}} J(t, \mathbf{z}(t), \mathbf{u}(t)),
$$

subject to

$$
\begin{aligned}
& \dot{\mathbf{z}}(\tau)=\mathbf{f}(\tau, \mathbf{z}(\tau), \mathbf{u}(\tau)), \\
& \mathbf{u}(\tau) \in U, \\
& \tau \in[t, t+T], \\
& \mathbf{z}(t+T) \in \Omega,
\end{aligned}
$$

where $\Omega$ is the terminal state region.

A terminal state controller $\tilde{\mathbf{u}}(\tau, \mathbf{z}(t))$ working in the terminal state region $\Omega$ needs to be constructed for $\tau>t+T$ to guarantee the feasibility of solving the OP problem at each time step. The details of how to find the terminal state region and terminal state controller for the formation control will be given in the next section. The MPC control algorithm can be described as follows:

Initialization Given initial state $\mathbf{z}(0)$ and $t=0$. A constructed control function is initialized $\hat{\mathbf{u}}(\tau, \mathbf{z}(0))=$ $0, \tau \in[0, T]$.

Open Loop Optimization The open loop optimal control function $\overline{\mathbf{u}}(\tau, \mathbf{z}(t))$ can be found by solving the OPs (10) and (11) for the period $0 \leq \tau \leq T$ using $\hat{\mathbf{u}}(\tau, \mathbf{z}(t))$ as the initial solution.

Feedback Control The MPC uses only $\overline{\mathbf{u}}(\tau, \mathbf{z}(t))$ to control the system (8) over a period $\tau \in[t, t+\delta)$. The open loop control function $\overline{\mathbf{u}}(\tau, \mathbf{z}(t))$ defines a state trajectory $\overline{\mathbf{z}}(\tau, \mathbf{z}(t))$. Both of them depends on the current state $\mathbf{z}(t)$. Therefore, the MPC control function $\mathbf{u}(\tau, \mathbf{z}(t))$ and the corresponding state trajectory $\mathbf{z}(\tau, \mathbf{z}(t))$ are

$$
\begin{aligned}
& \mathbf{u}(\tau, \mathbf{z}(t))=\overline{\mathbf{u}}(\tau, \mathbf{z}(t)), \\
& \mathbf{z}(\tau, \mathbf{z}(t))=\overline{\mathbf{z}}(\tau, \mathbf{z}(t)), \quad \tau \in[t, t+\delta) .
\end{aligned}
$$

Feasible Control Construction A terminal state controller $\tilde{\mathbf{u}}(\tau, \mathbf{z}(t+\delta))$ for the terminal state region $\Omega$ is found first. Then, a feasible control function $\hat{\mathbf{u}}(\tau, \mathbf{z}(t+\delta))$ is constructed based on the open loop optimal control function $\overline{\mathbf{u}}(\tau, \mathbf{z}(t+\delta))$ and the terminal state controller $\tilde{\mathbf{u}}(\tau, \mathbf{z}(t+\delta))$ :

$$
\begin{aligned}
& \hat{\mathbf{u}}(\tau, \mathbf{z}(t+\delta)) \\
& \quad=\left\{\begin{array}{c}
\overline{\mathbf{u}}(\tau, \mathbf{z}(t+\delta)), t+\delta \leq \tau<t+T \\
\tilde{\mathbf{u}}(\tau, \mathbf{z}(t+\delta)), t+T \leq \tau<t+T+\delta
\end{array}\right.
\end{aligned}
$$

Continue $t \leftarrow t+\delta$ and go to the Open Loop Optimization step.

In the whole control process, the control signal $\mathbf{u}(\tau, \mathbf{z}(t))$ depends on the current state $\mathbf{z}(t)$ at each step. Therefore, it is a state feedback controller.

Suppose the leader speed signals are lower bounded and the OP is feasible at time $t=0$. The MPC control algorithm described above for the system (8) is asymptotically stable if there exists a terminal state controller $\tilde{\mathbf{u}}(\tau, \mathbf{z}(t))$ such that the following condition is satisfied:

$$
\dot{g}(\mathbf{z}(t))+L(t, \mathbf{z}(t), \mathbf{u}(t)) \leq 0, \quad \tilde{\mathbf{u}}(\tau, \mathbf{z}(t)) \in U
$$

for any state $\mathbf{z}(t)$ belonging to the terminal region $\Omega$. This result has been used for a robot regulation problem in ref. [29]. Its proof can be found there.

\section{Terminal State Regions and Controllers}

A Lyapunov-like function is defined as the terminal state penalty term for the formation control purpose:

$$
g(\mathbf{z}(t+T))=\frac{1}{2} \mathbf{z}(t+T)^{T} S \mathbf{z}(t+T),
$$

where $S$ is a positive definite symmetric weight matrix. 
As the stability theorem stated, the terminal state regions and controllers should be designed to meet the inequality (14). The terminal state controllers are virtual controllers because they are used to find the terminal state region for the OP and never used for practical control. The analysis is discussed for $l-\phi$ control and $l-l$ control separately below.

\subsection{The $l-\phi$ control}

We define a terminal state controller for $l-\phi$ control as follows:

$$
\tilde{\mathbf{u}}_{j}=G_{j}^{-1} K_{j} \mathbf{z}_{j},
$$

where $K_{j}=\operatorname{diag}\left[k_{i}\right], k_{i}>0(i=1,2)$. As it is a virtual controller, we can select $k_{i}$ small enough to satisfy the speed saturation constraints. For example, we can use $k_{1}=k_{2}$ and let $u_{j \max }$ denote the limit of $\mathbf{u}_{j}$. Due to $G_{j} \tilde{\mathbf{u}}_{\mathbf{j}}=k_{1} \mathbf{z}_{j}$, we know $k_{i} \leq u_{j \max }\left\|G_{j}\right\| /\left\|\mathbf{z}_{j}\right\|$.

Combining Eqs. (3), (14), (15), and (16), we have,

$$
\begin{aligned}
\dot{g}\left(\mathbf{z}_{j}(t+T)\right)+L\left(t+T, \mathbf{z}_{j}(t+T), \mathbf{u}_{j}(t+T)\right) \\
=\mathbf{z}_{j}(t+T)^{T} S F_{j} \mathbf{u}_{i}(t+T)+\mathbf{z}_{j}(t+T)^{T} \\
\quad \times\left(P-S K_{j}\right) \mathbf{z}_{j}(t+T) .
\end{aligned}
$$

By selecting

$$
P-\frac{1}{2} S K_{j}-\frac{1}{2} K_{j} S \leq 0
$$

the stability condition (14) becomes

$$
\mathbf{z}_{j}(t+T)^{T} S F_{j} \mathbf{u}_{i}(t+T) \leq 0
$$

where $S=\operatorname{diag}\left[s_{i}\right], \quad s_{i} \geq 0 \quad(i=1,2)$. Equation becomes

$$
s_{1} \tilde{l}_{i j} \cos \psi_{i j} v_{i}-s_{2} \frac{\sin \psi_{i j}}{l_{i j}} v_{i} \tilde{\psi}_{i j}+s_{2} \tilde{\psi}_{i j} w_{i} \leq 0 .
$$

In the formation control, we know $l_{i j} \geq 0$ and assume $v_{i} \geq 0$, so the terminal state region $\Omega$ can be designed as follows to meet the conditions in Eq. (20):

$$
\begin{aligned}
\tilde{l}_{i j} \cos \psi_{i j} & \leq 0, \\
\tilde{\psi}_{i j} \sin \psi_{i j} & \geq 0, \\
\tilde{\psi}_{i j} w_{i} & \leq 0 .
\end{aligned}
$$

\subsection{The $l-l$ control}

We define a terminal state controller for $l-l$ control as follows:

$$
\tilde{\mathbf{u}}_{j k}=G_{j k}^{-1} K_{j k} \mathbf{z}_{j k},
$$

where $K_{j k}=\operatorname{diag}\left[k_{i}\right], k_{i}>0(i=1,2,3,4)$. As it is a virtual controller, we can select $k_{i}$ small enough to satisfy the speed saturation constraints. For example, we can use $k_{1}=k_{2}=k_{3}=k_{4}$ and let $u_{j k \max }$ denote the limit of $\mathbf{u}_{j k}$. Due to $G_{j k} \tilde{\mathbf{u}}_{\mathbf{j k}}=k_{1} \mathbf{z}_{j k}$, we know $k_{i} \leq u_{j k \max }\left\|G_{j k}\right\| /\left\|\mathbf{z}_{j k}\right\|$.
Combining Eqs. (6), (14), (15), and (22), we have

$$
\begin{aligned}
\dot{g}\left(\mathbf{z}_{j k}(t+T)\right)+L\left(t+T, \mathbf{z}_{j k}(t+T), \mathbf{u}_{j k}(t+T)\right) \\
=\mathbf{z}_{j k}(t+T)^{T} S F_{j k} \mathbf{u}_{i}(t+T)+\mathbf{z}_{j k}(t+T)^{T} \\
\quad \times\left(P-S K_{j k}\right) \mathbf{z}_{j k}(t+T) .
\end{aligned}
$$

By selecting

$$
P-\frac{1}{2} S K_{j k}-\frac{1}{2} K_{j k} S \leq 0,
$$

the stability condition (19) becomes

$$
\mathbf{z}_{j k}(t+T)^{T} S F_{j k} \mathbf{u}_{i}(t+T) \leq 0,
$$

or

$$
\begin{aligned}
& s_{1} \tilde{l}_{i j} \cos \psi_{i j} v_{i}-s_{2} \frac{\sin \psi_{i j}}{l_{i j}} v_{i} \tilde{\psi}_{i j}+s_{2} \tilde{\psi}_{i j} w_{i} \\
& \quad+s_{3} \tilde{l}_{i k} v_{i} \cos \psi_{i k} \leq 0 .
\end{aligned}
$$

In the formation control, we know $l_{i j} \geq 0, l_{i k} \geq 0$ and assume $v_{i} \geq 0$. By selecting $s_{1}=s_{3}$, the terminal state region $\Omega$ can be designed as follows to meet the conditions in Eq. (26):

$$
\begin{aligned}
\tilde{l}_{i j} \cos \psi_{i j}+\tilde{l}_{i k} \cos \psi_{i k} & \leq 0, \\
\tilde{\psi}_{i j} \sin \psi_{i j} & \geq 0, \\
\tilde{\psi}_{i j} w_{i} & \leq 0 .
\end{aligned}
$$

The internal states $\phi_{i j}$ in $l-\phi$ control with the controller (16) or $\theta_{j}$ and $\theta_{k}$ in $l-l$ control with the controller (22) are asymptotically stable (see ref. [27] for details).

\section{Simulation Results}

The simulation is carried out in MATLAB. The predictive control horizon $T$ and the control time interval $\delta$ are selected as $T=2 \mathrm{~s}$ and $\delta=0.2 \mathrm{~s}$. The maximum number of the OPs (10) and (11) is selected as 5 for the period $0 \leq \tau \leq T$. The leader trajectory is a circle,

$$
\begin{aligned}
& x_{i}=0.8 \cos (0.5 t), \\
& y_{i}=0.8 \sin (0.5 t),
\end{aligned}
$$

or a sinusoid curve,

$$
\begin{aligned}
& x_{i}=t / 5+\pi / 5, \\
& y_{i}=\sin (t / 5+\pi / 5) .
\end{aligned}
$$

The weight parameters of the cost function are selected as

$$
p_{i}=0.5, s_{i}=1, k_{i}=0.8 \quad(i=1,2,3,4) .
$$




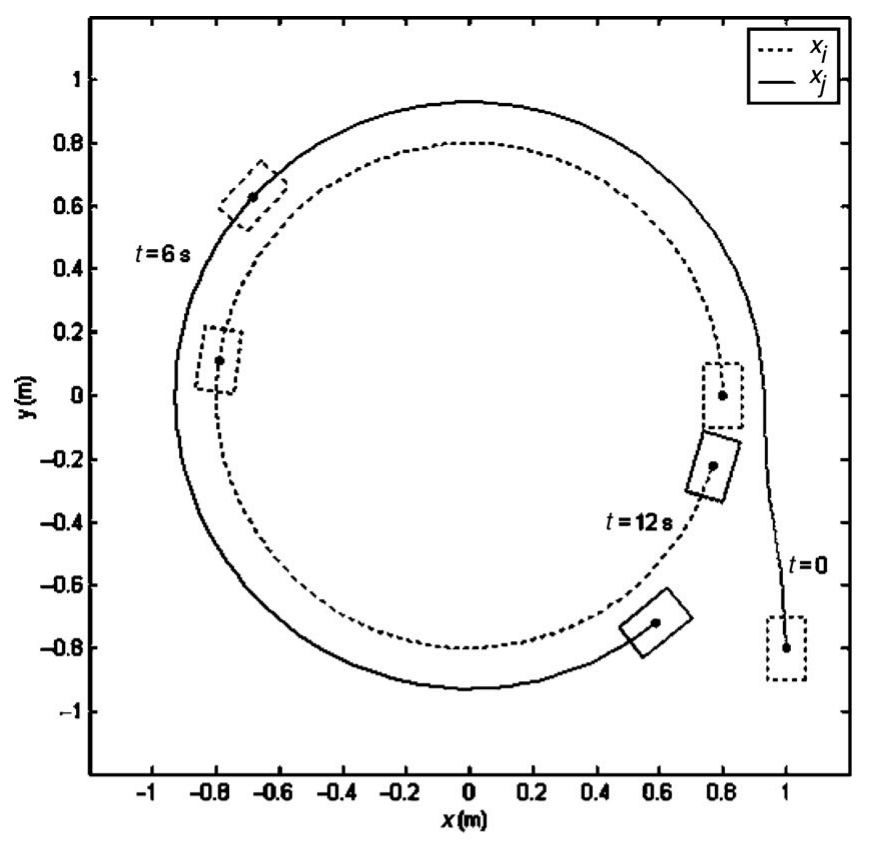

(a) Formation trajectories

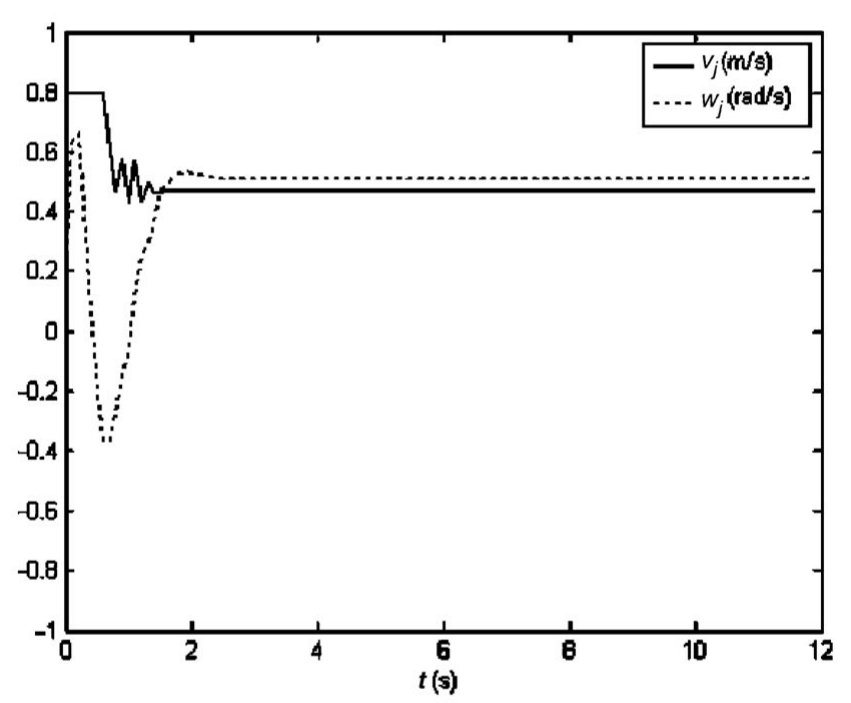

(b) Speed control signals

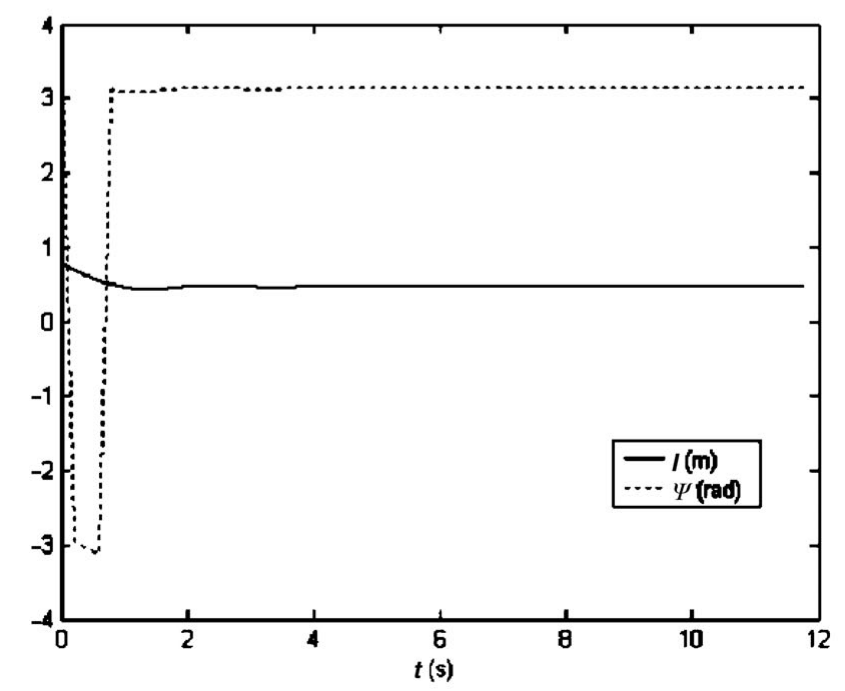

(c) Formation states

Fig. 2. The $l-\phi$ control in a circle test with initial position $(1,-0.8, \pi / 2)$.
The control signal constraints are the saturated constraints

$$
\begin{array}{r}
0 \leq v \leq v_{\max }, \\
-w_{\max } \leq w \leq w_{\max } .
\end{array}
$$

They are selected as

$$
\begin{aligned}
v_{\max } & =0.8(\mathrm{~m} / \mathrm{s}) \\
w_{\max } & =\pi / 2(\mathrm{rad} / \mathrm{s})
\end{aligned}
$$

\subsection{The $l-\phi$ control}

At first, the follower was initially positioned at $(1,-0.8, \pi / 2)$ and its speeds began with $(0,0)$ in a circle test. The desired formation pattern was $\left[l_{i j}^{d}, \psi_{i j}^{d}\right]=[0.5, \pi]$. The control results are shown in Fig. 2(a) for the trajectories, in Fig. 2(b) for the speed control signals, and in Fig. 2(c) for the formation states. From the trajectories, it can be seen that the follower

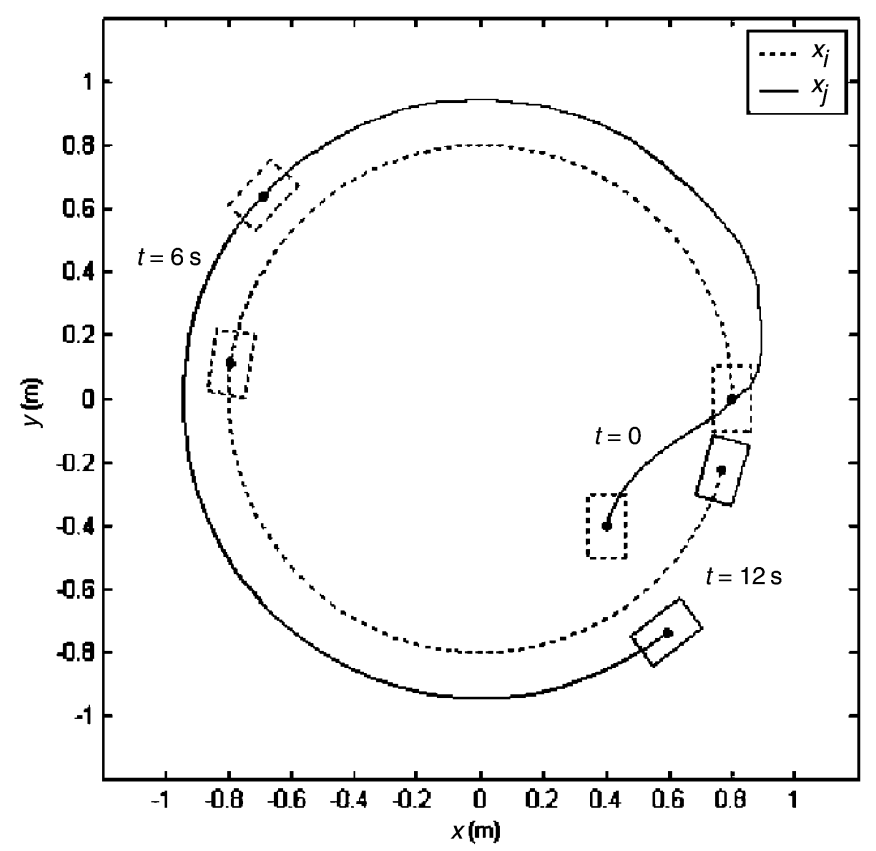

Fig. 3. The $l-\phi$ control in a circle test with initial position $(0.4,-0.4, \pi / 2)$.

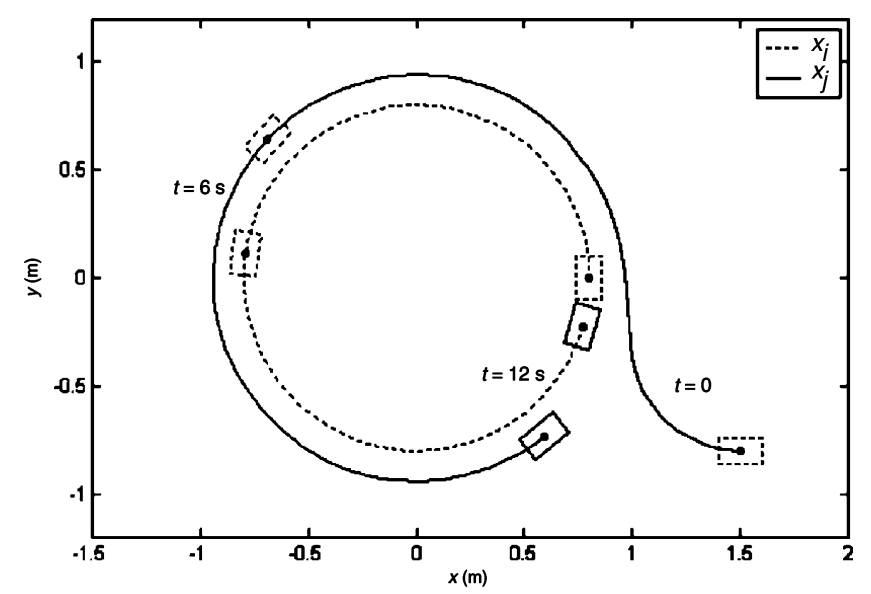

Fig. 4. The $l-\phi$ control in a circle test with initial position $(1.5,-0.8, \pi)$. 


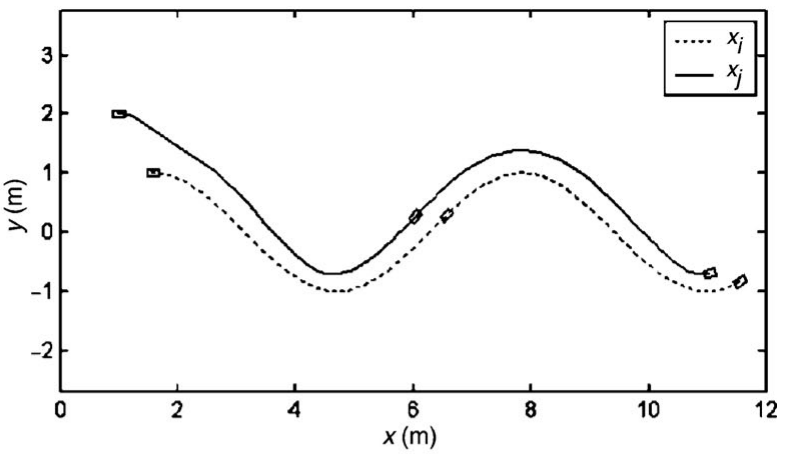

(a) Formation trajectories

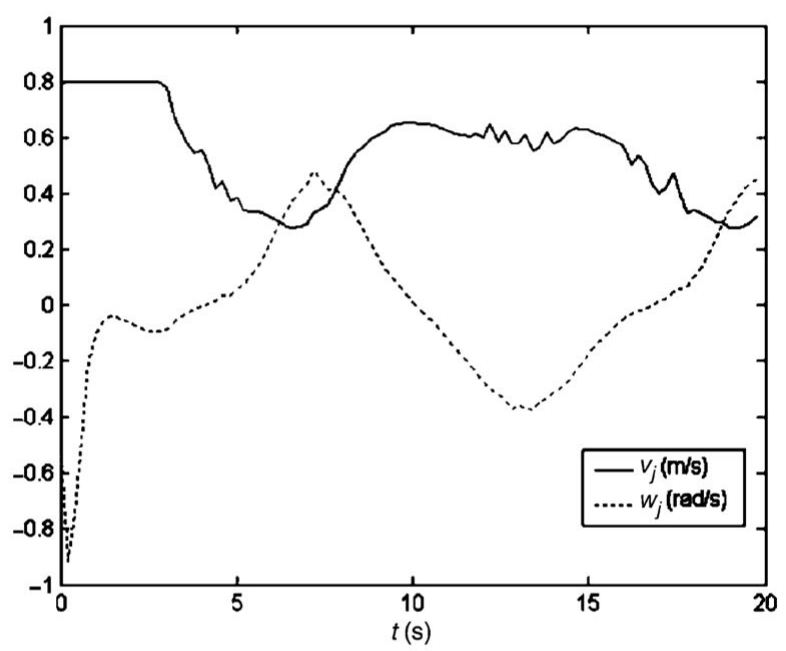

(b) Speed control signals

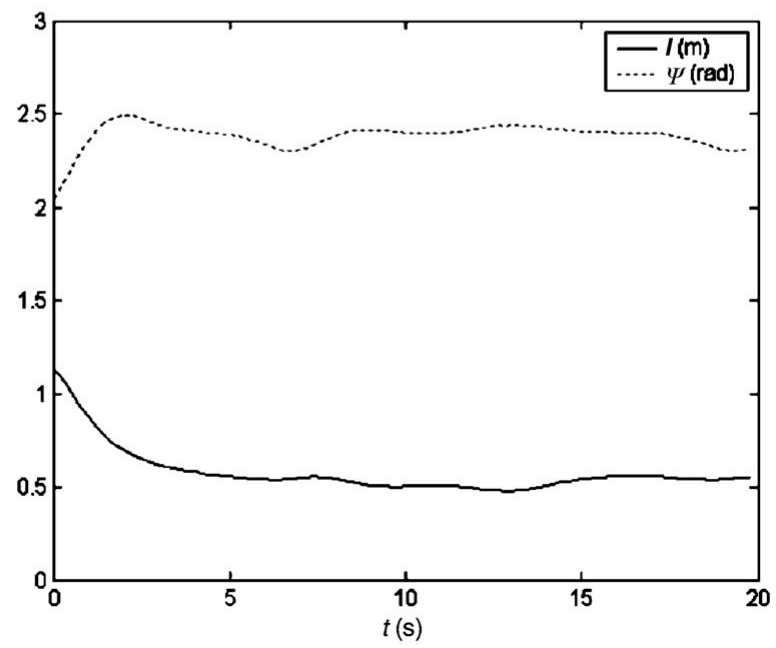

(c) Formation states

Fig. 5. The $l-\phi$ control in a sinusoid test with initial position $(1,2,0)$.

can adjust itself to form the desired formation pattern. The stable formation pattern can be checked in Fig. 2(c) where the formation states $l$ and $\phi$ gradually achieved their desired values. The speed control signals are shown in Fig. 2(b). After a period of transient time, the speed control signals finally converged to their stable values and they did not exceed their saturation constraints.

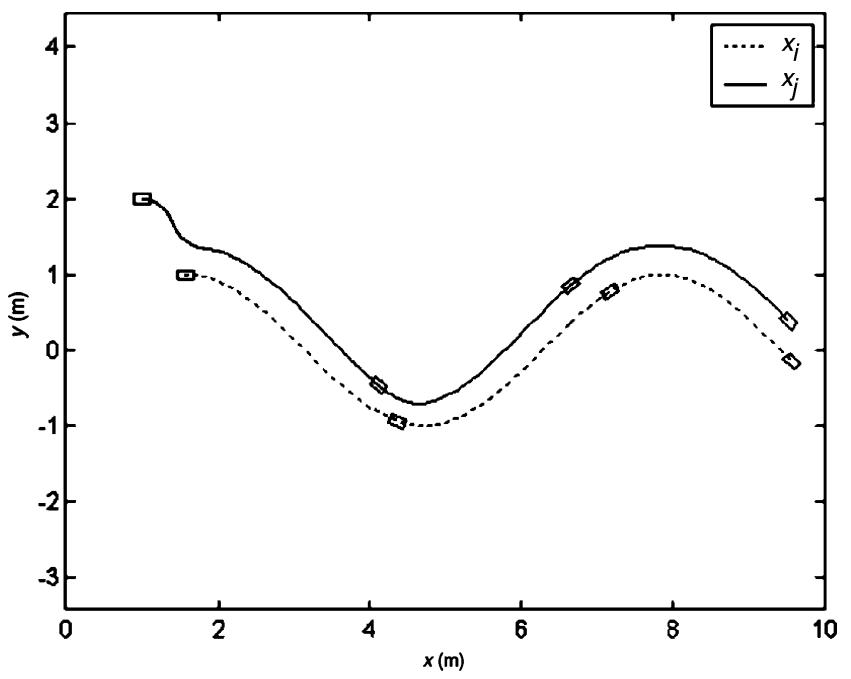

Fig. 6. The $l-\phi$ control in a sinusoid test with short control horizon.

For the same leader's circle trajectory, the follower started from different initial positions to further verify the MPC controller. The initial positions were $(0.4,-0.4, \pi / 2)$ and $(1.5,-0.8, \pi)$. The same control parameters and formation pattern were used. The results are shown in Figs. 3 and 4. The followers in both tests caught up with the leader and gradually formed the desired formation pattern.

Next, the leader moved along a sinusoid curve and the desired formation pattern was $\left[l_{i j}^{d}, \psi_{i j}^{d}\right]=[0.5,3 \pi / 4]$. The initial position of the follower was selected as $(1,2,0)$ and its speed began with $(0,0)$. The control results are shown in Fig. 5(a) for the trajectories, in Fig. 5(b) for the speed control signals, and in Fig. 5(c) for the formation states. The trajectories and the formation states show that the desired formation pattern was finally formed. And the speed control signals did not exceed their limitations.

Using the MPC controller can adjust the formation performance by changing the controller's parameters. In Fig. 6 , the predictive control horizon $T$ and the control time interval $\delta$ were selected as $T=1 \mathrm{~s}$ and $\delta=0.1 \mathrm{~s}$. All other parameters were the same as in Fig. 5. It means the follower in Fig. 6 has a shorter view (shorter prediction time) than in Fig. 5. Therefore, the follower moved quickly to the leader. This can be observed at the beginning of the trajectory in Fig. 6. It should be noted that the small horizon does not always lead to the quick move to the leader. The main reasons for this application are that in the receding horizon cost, no control weighting is employed and the terminal penalty is quite large.

\subsection{The $l-l$ control}

A circular leader was tested first. The desired formation pattern was $\left[l_{i j}^{d}, \psi_{i j}^{d}, l_{i k}^{d}, \psi_{i k}^{d}\right]=[0.5,3 \pi / 4,0.5,0.5 \sqrt{2}]$. Two followers $R_{j}$ and $R_{k}$ were initially positioned at $(0.8,-0.4, \pi / 2)$ and $(0.8,-0.8, \pi / 2)$. The control results are shown in Fig. 7(a) for the trajectories, in Fig. 7(b) for the control signals, and in Fig. 7(c) for the formation states. The trajectories and formation states show that the desired formation pattern was finally formed. The speed 


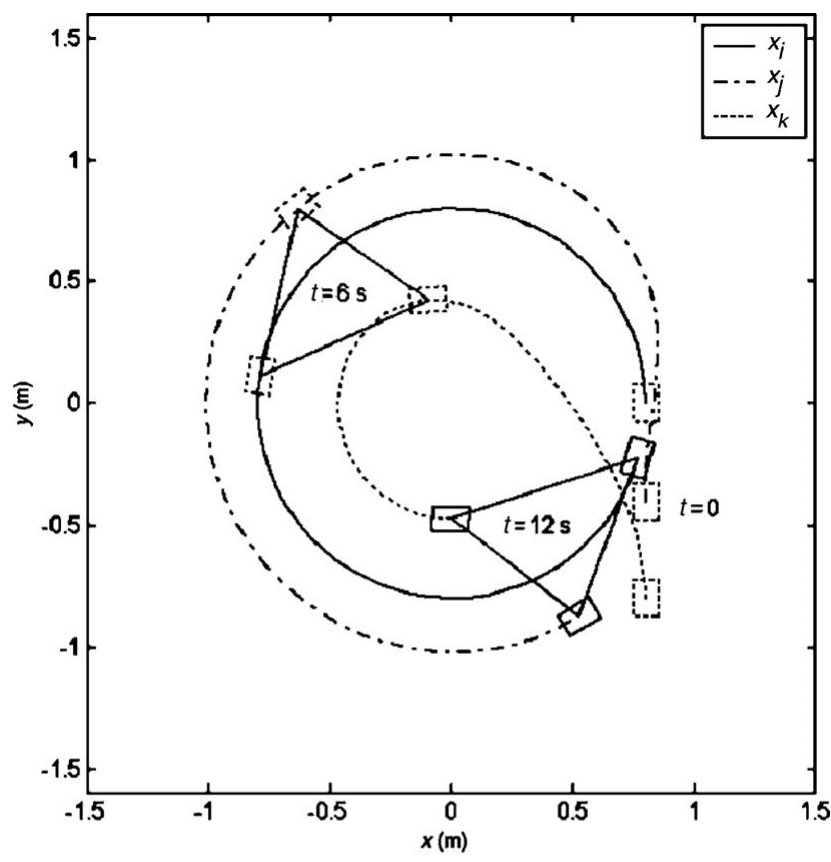

(a) Formation trajectories

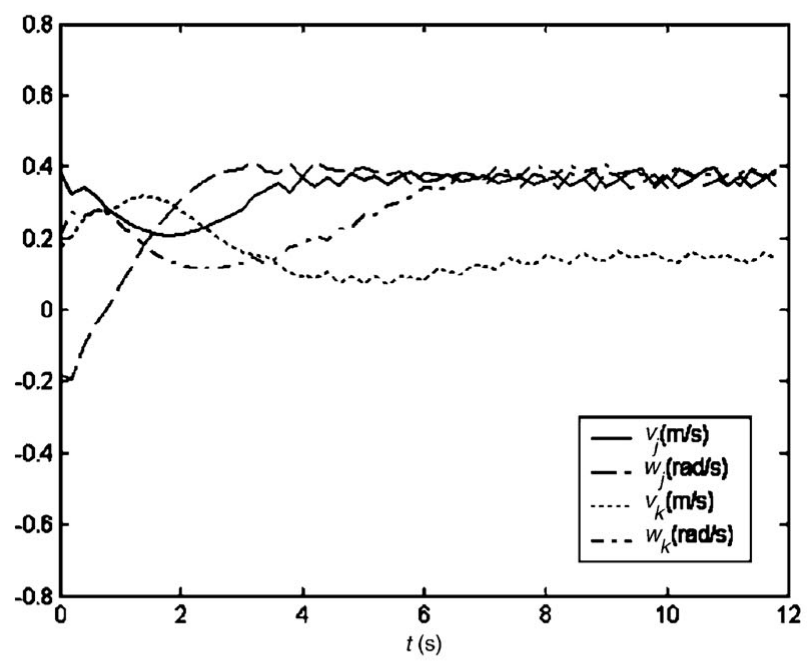

(b) Speed control signals

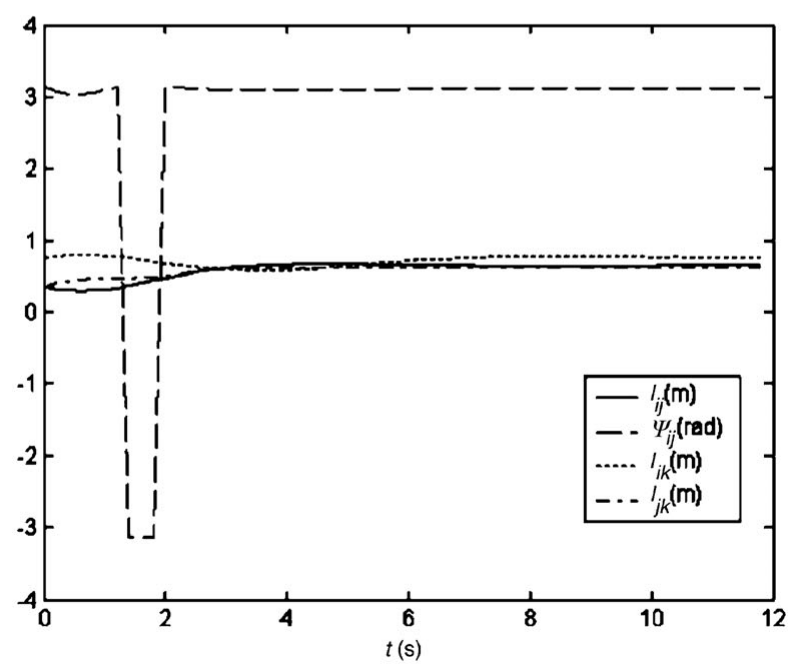

(c) Formation states

Fig. 7. The $l-l$ control in a circle test.

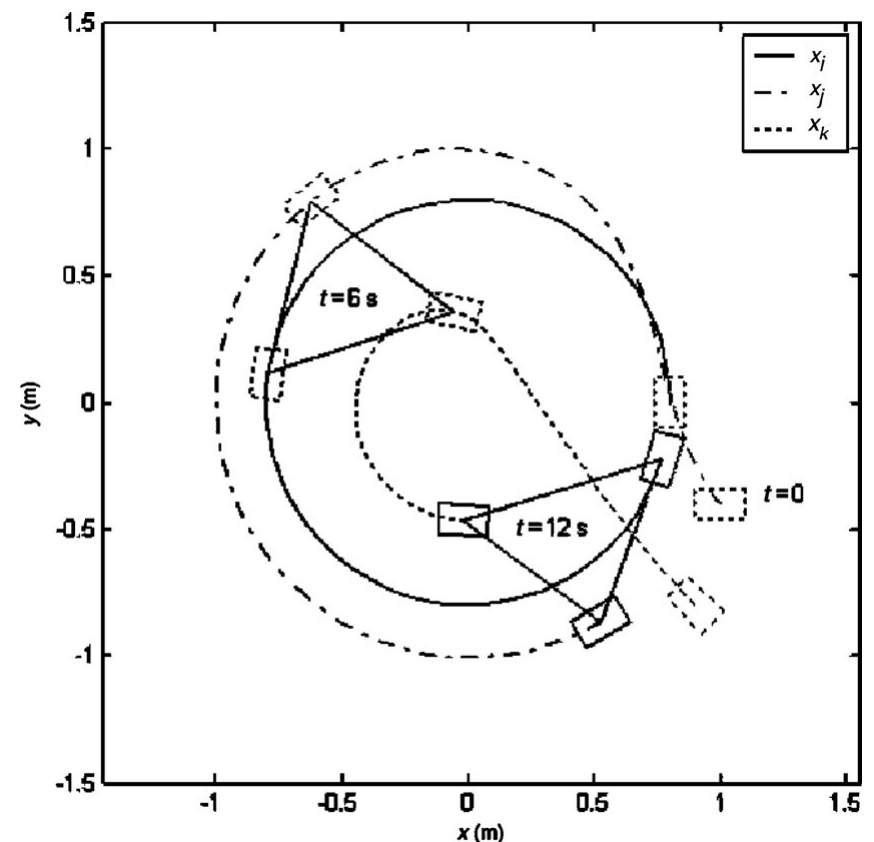

Fig. 8. The $l-l$ control in a circle test with different initial positions.

control signals fell within their limitations. Another test in Fig. 8 confirmed the formation results where the followers $R_{j}$ and $R_{k}$ were initially positioned at $(1,-0.4, \pi)$ and $(0.9,-0.8,3 \pi / 4)$.

In the sinusoid tests, the followers were initially positioned at $(0.5,1,0)$ and $(1,0, \pi / 2)$. The control results are shown in Fig. 9(a) for the trajectories, in Fig. 9(b) for the speed control signals, and in Fig. 9(c) for the formation states. The followers gradually moved to form the desired formation pattern. The stable formation states can be seen in Fig. 9(c). The speed control signals were displayed in Fig. 9(b) and they did not exceed their limitations.

\section{Conclusions}

This paper proposes an MPC control scheme for robots to follow a virtual leader. Two models are used: $l-\phi$ control and $l-l$ control. The terminal state controllers are developed and new terminal state regions are found. By using the terminal state regions as the constraints in the OP of the MPC controller, the control stability is guaranteed.

The use of an MPC can solve constrained control problems and optimize the control performance. In this paper, the input saturation constraint is treated. The state constraint could also be included to handle the collision avoiding problems in our next step work. The performance optimization should be balanced with the computation time reducing. It has been shown that the suboptimal solution (obtained by several optimization iterations from a feasible solution) can also guarantee the MPC stability. ${ }^{24,29}$ The longer the optimization time, the better the control performance. However, a long computation time is not achievable for a real-time control. For practical use, hardware implementation of an MPC is a possible option. ${ }^{30}$ 


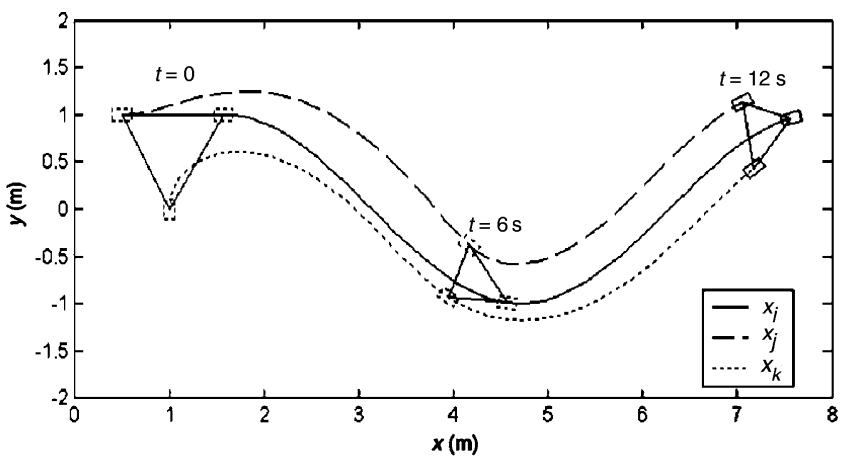

(a) Formation trajectories

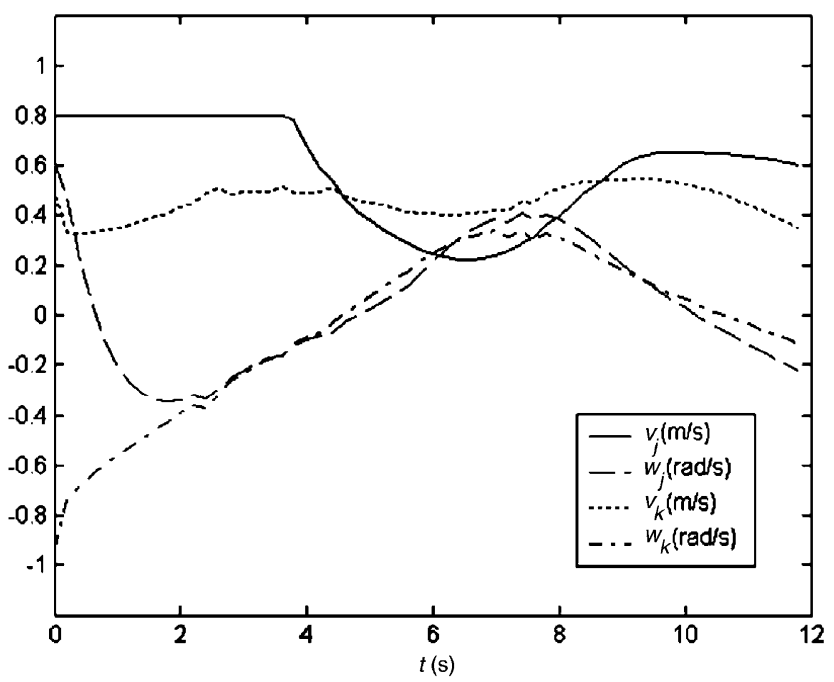

(b) Speed control signals

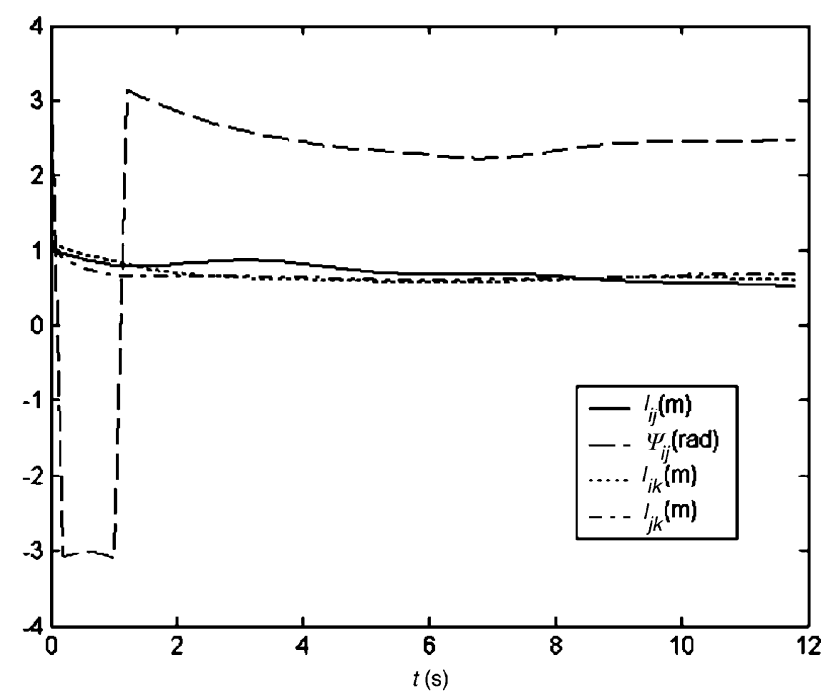

(c) Formation states

Fig. 9. The $l-l$ control in a sinusoid test.

\section{References}

1. R. M. Murray, "Recent research in cooperative control of multivehicle systems," J. Dyn. Syst. Meas. Control 129(5), 571-583 (2007).

2. H. Tanner and D. Christodoulakis, "Decentralized cooperative control of heterogeneous vehicle groups," Robot. Auton. Syst. 55(11), 811-823 (2007).
3. M. A. Hsieh, S. Loizou and V. Kumar, "Stabilization of multiple robots on stable orbits via local sensing," Proceedings of IEEE Conference on Robotics and Automation (Apr. 10-14, 2007).

4. M. Zavlanos and G. J. Pappas, "Distributed formation control with permutation symmetries," Proceedings of IEEE Conference on Decision and Control, New Orleans, LA (Dec. 2007).

5. J. Finke and K. M. Passino, "Stable cooperative vehicle distributions for surveillance," ASME J. Dyn. Syst. Meas. Control 129, 597-608 (2007).

6. T. Balch and R. Arkin, "Behavior-based formation control for multi-robot teams," IEEE Trans. Robot. Autom. 14(6), 926-939 (1998).

7. J. Fredslund and M. Mataric, "A general algorithm for robot formations using local sensing and minimal communication," IEEE Trans. Robot. Autom. 18(5), 837-846 (2002).

8. F. Giulietti, L. Pollini and M. Innocenti, "Autonomous formation flight," IEEE Control Syst. Mag. 18(5), 34-44 (Dec. 2000).

9. R. Vidal, O. Shakernia and S. Sastry, "Formation control of nonholonomic mobile robots with omnidirectional visual servoing and motion segmentation," Proceedings of IEEE Conference on Robotics and Automation. (May 2003).

10. M. Egerstdt and X. Hu, "Formation constrained multi-agent control," IEEE Trans. Robot. Autom. 17(6), 947-951 (2001).

11. R. Fierro, A. Das, V. Kumar and J. Ostrowski, "Hybrid control of formation of robots," Proceedings of the 2001 IEEE ICRA, Korea (May 21-26, 2001) pp. 157-162.

12. H. Tanner, G. Pappas and V. Kumar, "Leader-to-formation stability," IEEE Trans. Robot. Autom. 20(3), 433-455 (2004).

13. J. Desai, J. Ostowski and V. Kumar, "Modeling and control of formations of nonholonomic mobile robots," IEEE Trans. Robot. Autom. 17(6), 905-908 (2001).

14. J. Lawton, R. Beard and B. Young, "A decentralized approach to formation manoeuvres," IEEE Trans. Robot. Autom. 19(6), 933-941 (2003)

15. M. Egerstdt and X. Hu, "A control Lyapunov function approach to multi-agent coordination," IEEE Trans. Robot. Autom. 18(5), 847-851 (2002).

16. S. S. Keerthi and E. G. Gilbert, "Optimal infinite-horizon feedback laws for a general class of constrained discretetime systems: stability and moving-horizon approximations," J. Optim. Theory Appl. 57, 265-293 (1988).

17. J. B. Rawlings and K. R. Muske, "Stability of constrained receding horizon control," IEEE Trans. Autom. Control 38(10), 1512-1516 (1993).

18. H. Chen and F. Allgower, "A quasi-infinite horizon nonlinear model predictive control scheme with guaranteed stability," Automatica 34(10), 1205-1217 (1998).

19. G. D. Nicolao, L. Magni and R. Scattolini, "Stabilizing receding-horizon control of nonlinear time varying systems," IEEE Trans. Autom. Control 43, 1030-1036 (1998).

20. F. A. C. C. Fontes, "A general framework to design stabilizing," Syst. Control Lett. 42(2), 127-143 (2001).

21. A. Jadbabaie, J. Yu and J. Hauser, "Stabilizing receding horizon control of nonlinear systems: A control Lyapunov function approach," Proceedings of American Control Conference, San Diego, CA (1999).

22. D. Q. Mayne, J. B. Rawlings, C. Rao and P. Scokaert, "Constrained model predictive control: Stability and optimality," Automatica 36, 789-814 (2000).

23. J. Primbs, V. Nevistic and J. Doyle, "A receding horizon generalization of pointwise min-norm controllers," IEEE Trans. Autom. Control 45(5), 898-909 (2000).

24. P. Scokaert, D. Mayne and J. Rawlings, "Suboptimal model predictive control (feasibility implies stability)," IEEE Trans. Autom. Control 44, 648-654 (1999).

25. W. Dunbar and R. Murray, "Model predictive control of coordinated multi-vehicle formations," Automatica 2(4), 549558 (2006). 
26. K. Wesselowski and R. Fierro, "A dual-mode model predictive controller for robot formations," Proceedings of the 42nd IEEE $C D C$, Hawaii (2003) pp. 3615-3620.

27. A. Das, R. Fierro, V. Kumar, J. Ostrowski, J. Spletzer and C. Taylor, "A vision-based formation control framework," IEEE Trans. Robot. Autom. 18(5), 813-825 (2002).

28. H. Takahashi, H. Nishi and K. Ohnishi, "Autonomous decentralized control for formation of multiple mobile robots considering ability of robot," IEEE Trans. Ind. Electron. 51(6), 1272-1279 (2004).

29. D. Gu and $\mathrm{H}$. Hu, "A stabilizing receding horizon regulator for nonholonomic mobile robots," IEEE Trans. Robot. 21(5), 1022-1028 (2005).

30. L. Bleris, P. Vouzis, M. Arnold and M. Kothare, "A coprocessor FPGA platform for the implementation of real-time model predictive control," Proceedings of American Control Conference, Minneapolis, MN (June 2006). 